\title{
Classical Formulation of the Quantum Rayleigh Coupling of Photons
}

\author{
Andre Vatarescu \\ Fibre-Optic Transmission of Canberra, Batman Street 32, Canberra, ACT, Australia \\ Authore-mail address: andre_vatarescu@yahoo.com.au
}

\begin{abstract}
Despite multiple classical outcomes arising from the quantum Rayleigh conversions of photons underlying the propagation of optical waves through dielectric media and the ensuing light-matter interactions, this quantum process has been largely ignored. Several of its outcomes are considered in this article from a physical perspective, e.g., inter-quadrature coupling of photons, phase-dependent amplification in optical directional couplers and related polarization rotation, phase-shifting of weak signals in the optically linear regime, location-dependent coupling coefficient for refractive index gratings, etc. A correct identification of these effects will enable useful design and operation of integrated photonic functional devices.
\end{abstract}

Key words: Quantum Rayleigh emission; Phase-sensitive amplification; Integrated photonic devices.

\section{Introduction}

The widely used coupled-mode theory describing optical power coupling between two adjacent waveguides introduced in the 1960s - relies on a perturbation of the cladding as a physical mechanism. However, the gradient of the perturbed dielectric constant gives rise to a randomly scattering term in the comprehensive wave equation. This approach is inconsistent with directional coupling of photons and leads to physically impossible outcomes raising questions about the validity of its application as in recently published articles in the IEEE J. Quantum Electron., vol. 54, 2018 ( no. 1, article 6300206, and no. 2, article 6800207).

A physically meaningful and correct identification of processes underpinning the description of propagation and coupling of photons in dielectric media is crucial in order to open up new practical ways of designing, fabricating, and operating integrated photonic devices. The quantum Rayleigh conversion of photons (QRCP) [1, 2], provides a meaningful explanation for the operation of the optical directional couplers, in contrast to the perturbation approach, e.g. [3, 4], based on the fictional splitting of the dielectric constant $\varepsilon(x, y, z)=\varepsilon_{b}+\Delta \varepsilon$ into a uniform background $\varepsilon_{b}$ and a perturbation $\Delta \varepsilon(x, y, z)$ which is supposed to generate a coupling polarization $\Delta \boldsymbol{P}=\Delta \varepsilon \boldsymbol{E}$ induced by an overlaping optical field.

An optically linear parametric (OLP) interaction [5] consists of an electric dipole absorbing one photon and emitting one photon of the same frequency or energy, e.g. the elastic or Rayleigh scatterings $[1,2]$ associated with the QRCP. This quantum process is localized, can take place in both homogeneous and inhomogeneous dielectric media, and is described classically or macroscopically, by means of the interaction term included in the Poynting theorem [5] of the flow of energy $\nabla \cdot \boldsymbol{P}_{1}=-i \omega \boldsymbol{D}_{1} \cdot \boldsymbol{E}_{1}{ }^{*}-i \omega \boldsymbol{B}_{1} \cdot \boldsymbol{H}_{1}{ }^{*}-i \omega \boldsymbol{P}_{2} \cdot \boldsymbol{E}_{1}{ }^{*}$ with the common interpretation of the variables and constants in electromagnetic theory. These physical processes require that Maxwell's curl $\boldsymbol{H}$ equation of each wave be driven by the total electric dipole polarization available in the medium. The coupling term $\boldsymbol{P}_{2} \cdot \boldsymbol{E}_{1}{ }^{*}$ between one wave $\boldsymbol{E}_{1}{ }^{*}$ and the dipole polarization induced - through the linear susceptibility - by a second wave $\boldsymbol{P}_{2}=\chi \boldsymbol{E}_{2}$, and included in the equation of motion of the complex Poynting vector equation, would correspond to stimulated emission initiated by the spontaneous emission or another co-propagating wave of the same frequency [5].

The QRCP can couple photons between two optical waves propagating simultaneously through a homogeneous medium, i. e., for which $\Delta \varepsilon=0$. Two optical waves of the same frequency, co-propagating through an optically linear and homogeneous dielectric medium, can exchange photons and undergo mutually induced phase-shifts providing the possibility for a variety of integrated photonic devices such as phase-dependent amplification polarization-dependent amplification, optical directional couplers, X-junctions, Y-junctions, polarisation rotation with spontaneous emission, etc. 


\section{Equations of motion for the power and phase of interacting optical waves in a dielectric medium}

A physically meaningful coupled-wave formalism has been developed in the context of quantum Rayleigh conversion of photons for the operation of optical directional couplers, and other devices, in optically linear media as suggested in references [5-7], for both homogeneous and inhomogeneous dielectric configurations. The resultant rate equations for the exchange of optical power $P_{\mathrm{j}}(\mathrm{j}=1$ or 2$)$ between two interacting wavefronts and their corresponding phases $\varphi_{\mathrm{j}}$, have the following form [5-7]:

$$
\begin{gathered}
\frac{\partial}{\partial z} P_{1}=g_{1} P_{1} \\
g_{1}=-\kappa\left(P_{2} / P_{1}\right)^{1 / 2} \sin \theta_{21} \\
\frac{\partial}{\partial z} \theta_{21}=\Delta \beta+\kappa\left[\left(P_{1} / P_{2}\right)^{1 / 2}-\left(P_{2} / P_{1}\right)^{1 / 2}\right] \cos \theta_{21} \\
\frac{\partial}{\partial z} \varphi_{1}=\kappa\left(P_{2} / P_{1}\right)^{1 / 2} \cos \theta_{21} \\
\kappa=\left(k_{\mathrm{o}} / 2 n\right) \iint d x d y \chi^{(1)} f_{1} f_{2} \boldsymbol{e}_{1} \cdot \boldsymbol{e}_{2}
\end{gathered}
$$

where the gain coefficient $g$ includes a phase dependence and the coupling coefficient $\kappa$ is affected by the threedimensional polarization states of the two waves, $\boldsymbol{e}_{1}$ and $\boldsymbol{e}_{2}$. The phase difference between the two waves is $\theta_{21}=$ $\left(\beta_{2}-\beta_{1}\right) z+\varphi_{2}-\varphi_{1}, \beta$ being the propagation constant and $z / t=\mathrm{v}_{\mathrm{p}}$ is the phase velocity. In Eq. (5), $k_{\mathrm{o}}$ and $n$ specify the free-space wave vector and the effective refractive index, respectively. It should be noted that Eqs. (1-5) describe the physically meaningful process of quantum Rayleigh conversion of photons [5-7]. The coupling coefficient of Eq. (5) indicates that the entire local value of the optically linear susceptibility $\chi^{(1)}$ is involved in the coupling process inside the dielectric medium at any point where the two spatial distributions $f_{1}$ and $f_{2}$ overlap, each having units of $\mathrm{m}^{-1}$, and the squares $f^{2}$ being normalized to a dimensionless unit over the cross-section area. This is in contrast to the physically impossible coupling between two optical waveguides apparently induced by a perturbation of the dielectric constant $\Delta \varepsilon$ in the cladding which leads physically to random, classical Rayleigh scattering [8].

\section{Applications of the optically linear parametric interactions}

As a first application of equations (1-5) describing the physical process of quantum Rayleigh conversion of photons, we consider the optical directional coupler depicted in Fig. 1 as a cross-section of two waveguides of core indices $n_{1}$ and $n_{2}$ separated by a cladding of refractive index $n_{3}$. It is the overlap of modal fields in the cladding that brings about the exchange of optical power, with the coupling coefficient being proportional to the susceptibility $\chi_{3}$. The gain coefficient is phase- and polarisation- sensitive enabling selective amplification. Overall, it is possible to control the properties of a signal wave by adjusting the input values of a low-power pump wave because of the strong value of the optically linear susceptibility $\chi^{(1)}$. The relative phase (or phase-mismatch) can be controlled by adjusting the power ratio between the pump and the signal waves. Equations (3) and (5) indicate the existence of a built-in mechanism for phase-matching between a strong pump and a weak signal through the parametrically induced phase shift of Eq. (4). For converging or diverging Y-junctions, the coupling coefficient would have polarisation vectors projected onto each other.

By using the same optical frequency for an entire photonic circuit made up of dielectric waveguides, it should be possible to reduce the complexity associated with multiple waves and types of materials. Additionally, electro-optic waveguides provide connections between the electrical signals and optical ones [9]. 
The case of a refractive index grating incorporated into a waveguide as depicted in Fig. 2, would be of particular interest in order to couple power between two waves of different propagation constants, either co - propagating or counter-propagating. The susceptibility (or the refractive index $\mathrm{n}^{2}=\varepsilon / \varepsilon_{\mathrm{o}}=1+\chi$ ) becomes periodic $\chi(z)=$ $=\chi_{0}+\Delta \chi(z)$ leading to a $z$-dependent coupling coefficient $\kappa(z)$. The local coupling gain is maximized in Eqs. (2) and (5) above, for the longitudinal overlap between $\Delta \chi(z)$ and $\sin \theta_{21}$. For a sinusoidal variation $\Delta \chi=\sin (2 \pi z / \Lambda)$, the gain difference $\Delta g$ between the two halves of one periodic length $\Lambda$ and coupling direction over each half of $\Lambda$ is proportional to $\Delta g(z, \theta) \propto \Delta \chi \cdot \sin (2 \pi z / \Lambda) \cdot \sin (\Delta \beta \cdot z+\Delta \varphi)$ resulting in the phasematching condition $2 \pi / \Lambda=\Delta \beta+d(\Delta \varphi) / d z$ between the periodicity of $\chi$ and the relative phase $\theta=\Delta \beta \cdot z+\Delta \varphi$. A rectangular periodic perturbation of the susceptibility - see Fig. 2 - results in step-by-step amplification as the coupling in the first half of the period $\Lambda$, from one wave to the other is stronger than the opposite coupling over the second half of the period where $\Delta \chi=0$. We point out that the coupling coefficient varies with distance and is not the Fourier transform of the refractive index modulation function as suggested in [10, 11].

Another interesting application of equation (1-5) involves the optically linear parametric phase-pulling effect which shifts the phase of a weak signal towards a $(-\pi) / 2$ difference from that of strong pumps - see equations $(3)$ and (4). As a result, two optical waves co-propagating through a dielectric medium will have their photons transfer from one wave to the other depending on the relative phase between the two waves. This process, repeatedly, will eliminate optical waves whose phases diverge substantially from the phase of the surviving wave which will dominate the output and preserve the coherence of the optical beam despite light-matter interactions.

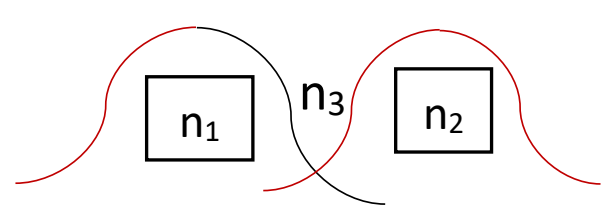

Fig. 1 The Rayleigh induced coupling of photons takes place in the cladding between the two waveguides with refractive index $\mathrm{n}_{3}$.

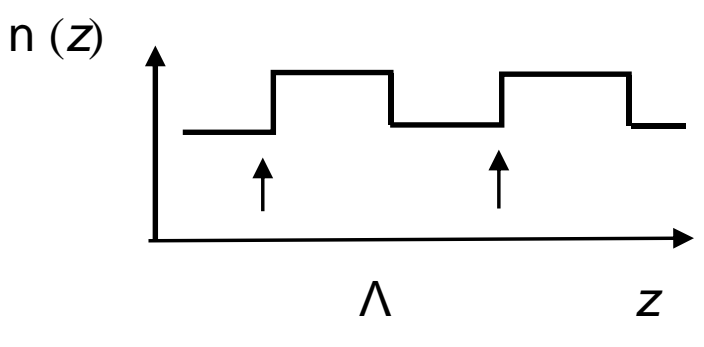

Fig. 2. The longitudinally varying coupling coefficient $\kappa(\mathrm{z})$ for an index grating.

\section{Conclusions}

Despite being presented in tens of textbooks and hundreds of published papers over half a century, the perturbation-based coupled-mode theory does not involve a physically meaningful process of stimulated emission which could be controlled in order to develop new photonic devices and applications. In contrast, the couple-wave analysis emerging from the quantum Rayleigh conversion of photons has the potential to operate with low levels of pump powers and delivers phase-sensitive and polarization- sensitive gain coefficients.

\section{References}

[1] W. H. Louisell, Quantum Statistical Properties of Radiation, John Wiley \& Sons , 1973.

[2] D. Marcuse, Principles of Quantum Electronics, Academic Press, 1980.

[3] B. Bai, L. Liu, and Z. Zhou, "Ultracompact, high extinction ratio polarization beam splitter-rotator based on hybrid plasmonic-dielectric directional coupling," Opt. Lett., 42, 4752 - 4755, (2017).

[4] G. P. Agrawal, Optical Waveguides; www2.optics.rochester.edu/users/gpa/opt468a.pdf.

[5] A. Vatarescu, "Photonic coupling between quadrature states of light in a homogeneous and optically linear dielectric medium,” J. Opt. Soc. Am. B, 31, 1741-1745, 2014. 
[6] A. Vatarescu, "Photonic Quantum Noise Reduction with Low-Pump Parametric Amplifiers for Photonic Integrated ircuits", Photonics, vol. 3, article 61, 2016.

[7] A. Vatarescu, "Phase-Sensitive Amplification with Low Pump Power for Integrated Photonics, "OSA Advanced Photonics Congress, paper ID: IM3A.6., 2016

[8] Z. Wang, H. Wu, X. Hu, N. Zhao, Qi Mo and \& G. Li, "Rayleigh scattering in few-mode optical fibers," Sci. Rep. 6, 35844; doi:10.1038/srep35844 (2016).

[9] A. Vatarescu, "Photonic quadrature-wave pulses generated by a single electro-optic waveguide modulator for digital transmission," J. Opt. Soc. Am. B, 32, 555-561, 2015.

[10] T. Erdogan, "Fiber Grating Spectra," J. Lightwave Technol., 15, 1277 -1294, 1997.

[11] L. Jin, W. Jin, J. Ju, and Y. Wang, “Coupled Local-Mode Theory for Strongly Modulated Long Period Gratings, “J. Lightwave Technol., 28, 1745-1751, 2010. 